\title{
Myocarditis in Children Requiring Critical Care Transport
}

\author{
Jordan S. Rettig and Gerhard K. Wolf \\ Additional information is available at the end of the chapter \\ http://dx.doi.org/10.5772/56177
}

\section{Introduction}

Myocarditis is an uncommon but potentially life-threatening presentation in pediatric patients requiring critical care transport. Patients may present with malignant arrhythmias and hemodynamic collapse, and may require transport to a center offering extracorporeal life support. In this chapter we aim to provide a brief overview of pediatric myocarditis, with a particular focus on considerations for stabilization and transport in acute fulminant myocarditis. These considerations include intubation and ventilation, hemodynamic support, induction of anesthesia and pharmacological considerations for sedation, patient triage, and choice of an appropriate receiving center.

\subsection{Etiology}

Myocarditis is an acute inflammatory disease of the myocardium, classically characterized by myocyte necrosis [1], which leads to ventricular dysfunction. There are several possible causes of myocarditis including infectious (viral, bacterial, fungal, yeast, parasitic, and protozoan) and non-infectious (immune mediated reactions, toxins, and other disorders). In many cases there is no identified cause. Most cases of pediatric myocarditis with a known etiology are caused by infections, in particular by viral infections [2]- [4], however a viral etiology may be difficult to detect. In a recent autopsy series examining 28 cases of myocarditis, viral analysis was done in 25 cases and was only positive in 9 of those. [5]

\section{Epidemiology and clinical presentation}

It has been estimated that pediatric cardiomyopathy occurs in between 1.13 and 1.24 per 100,000 patients, and more than $14 \%$ of these patients likely have cardiomyopathy from an 
infectious cause. [6]- [8] Klugman et al identified 216 cases of pediatric myocarditis over a oneyear period in 35 different children's hospitals, making up $0.05 \%$ of all patients seen. This group concluded that pediatric patients with myocarditis have considerable variability in their outcomes, use more intensive care unit (ICU) resources, and die more often than children with other diagnoses. [9] There is a broad range of clinical presentation ranging from asymptomatic to fulminant and symptoms are often non-specific. Some patients present with constitutional symptoms, and complaints of chest pain and fatigue are common. Additionally there may be large variability between presentations in different age groups. Patients with cardiac dysfunction may have syncope, heart failure, arrhythmias, or shock. [1] Fulminant myocarditis occurs in approximately $20-30 \%$ of all cases, and clinically presents with severe hemodynamic deterioration, cardiogenic shock, severe ventricular dysfunction, and possibly life-threatening arrhythmias. [10] Unlike adult patients, children more commonly present with fulminant myocarditis. [11] Myocarditis is a significant cause of sudden death and may result in the development of cardiomyopathy in some affected children. [12], [13]

\section{Diagnosis}

The diagnosis of myocarditis is often difficult. In one series of 31 cases of myocarditis in a pediatric emergency department, $57 \%$ of patients had been previously evaluated by a physician and diagnosed with pneumonia or asthma. [14] The less controversial diagnostic modalities include chest x-ray, electrocardiogram $(E K G)$ and echocardiogram. Sinus tachycardia on EKG with low-voltage QRS complexes is described as a classic finding. Beyond that there may be a variety of changes seen on EKG, including widened QRS complexes, non-specific ST changes, axis deviation, and/or Q waves. Patients may also present with arrhythmias including ventricular tachycardia, supraventricular tachycardia, and varying degrees of heart block.

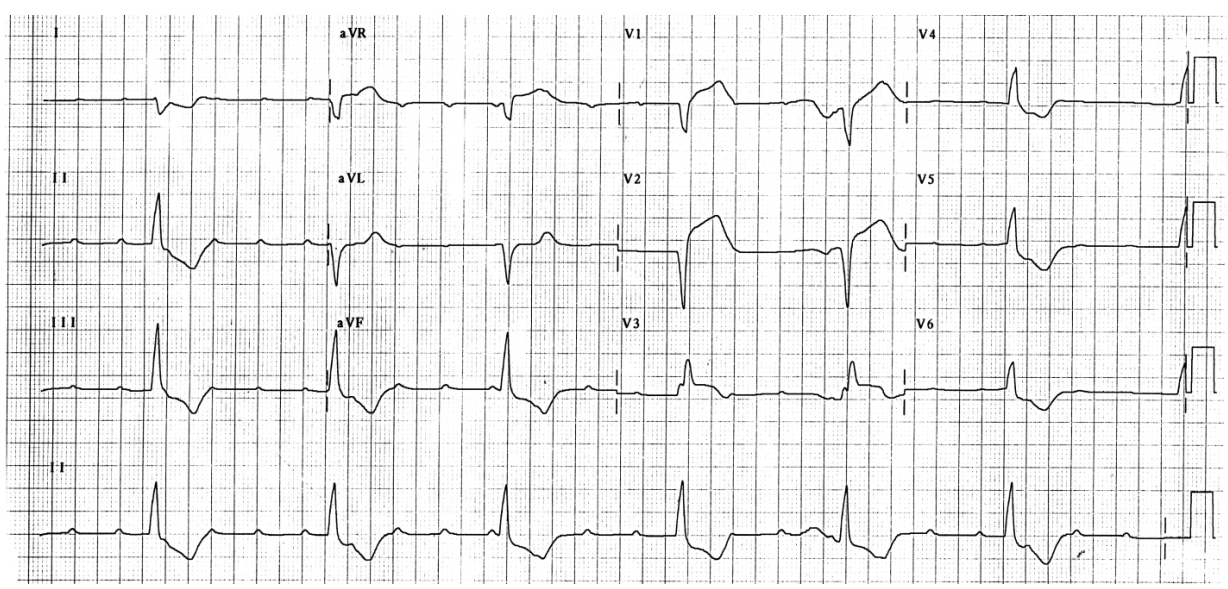

Figure 1. EKG of a 12 year old patient with myocarditis, atrioventricular block [15] 


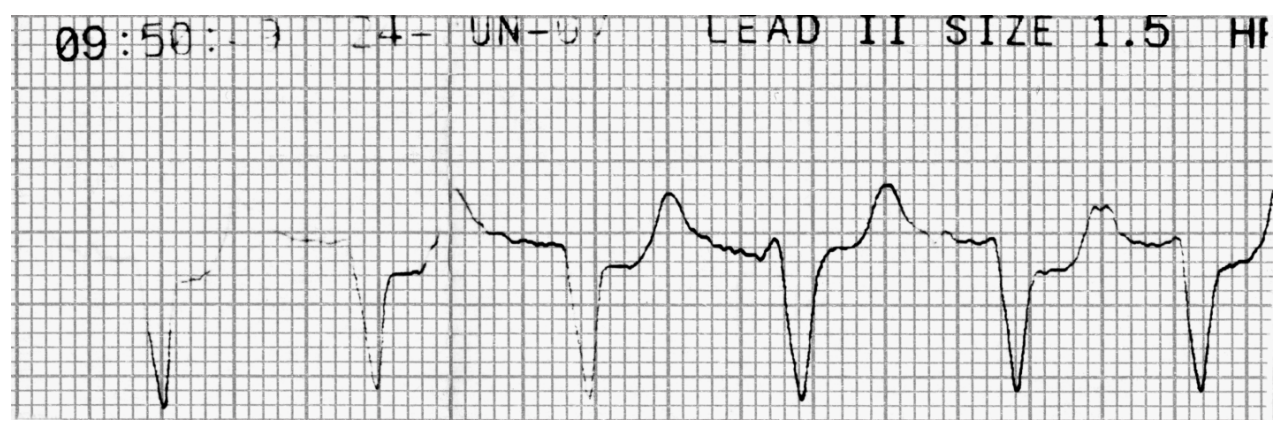

Figure 2. EKG (rhythm strip) of the same patient, who had ongoing severe ventricular dysfunction and developed intermittent episodes of wide-complex tachycardia [15]

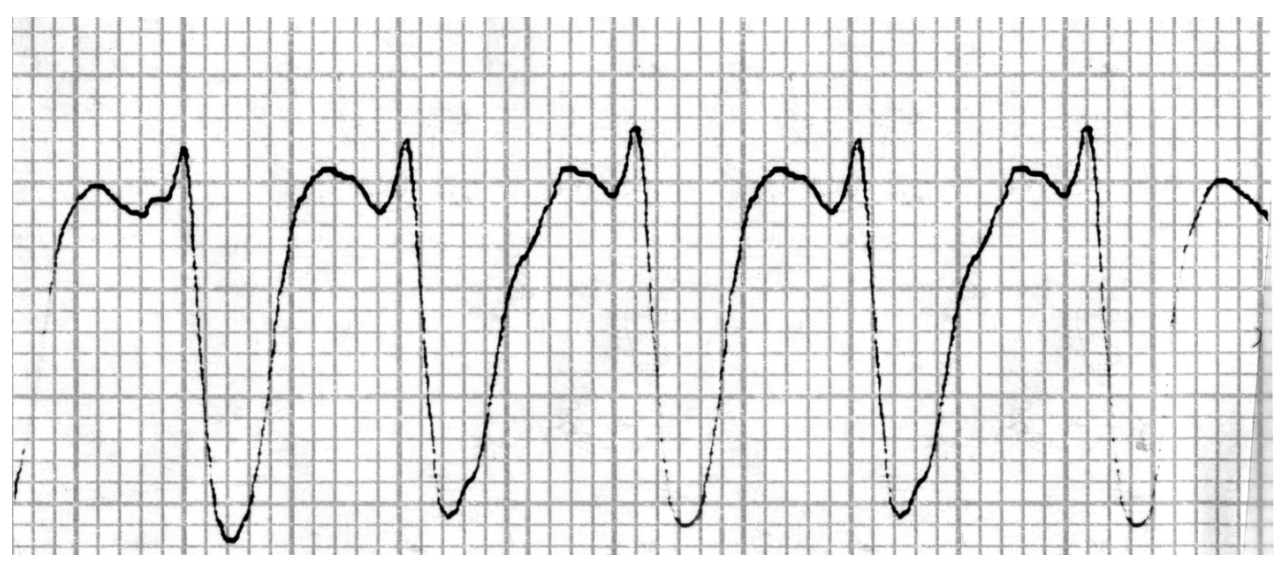

Figure 3. EKG (rhythm strip) of a 7 year old patient with myocarditis; wide-complex tachycardia [15]

Chest $\mathrm{x}$-ray findings tend to be consistent with congestive heart failure, including cardiomegaly and increased pulmonary markings suggestive of pulmonary edema. Echocardiography is a useful adjunct to assess ventricular dimensions, function, and presence of atrioventricular valve regurgitation or pericardial effusion.

A recent review of diagnostic strategies for myocarditis concluded that enlarged ventricular dimensions on echocardiography and elevated cardiac troponin levels were the most common findings. [16] Troponin I has high specificity but limited sensitivity in the diagnosis of myocarditis, despite the fact that it is otherwise a reliable and commonly available biomarker of myocardial injury. [17] In children, cardiac troponin T has been reported to have a sensitivity of $71 \%$ in myocarditis. [18] Other common laboratory studies include general markers of inflammation or infection, such as complete blood count with differential, C-reactive protein and erythrocyte sedimentation rate. It is also useful to examine markers of end organ perfusion 
including lactate, liver function tests and creatinine. These studies may help understand the etiology and impact of the disease process, but none are specific for myocarditis.

More controversial diagnostic modalities include cardiac magnetic resonance (CMR) imaging and endomyocardial biopsy (EMB). In general these techniques would not be employed in an acute setting in a non-tertiary care center. CMR has the advantage of being non-invasive it requires specialty equipment and radiologists familiar with the interpretation of findings. EMB is controversial for a variety of reasons, especially since it is invasive and carries a risk of adverse events. Also, myocardial inflammation tends to be patchy and may be missed by biopsy. A recent consensus statement by the American College of Cardiology and the European Society of Cardiology made a class IIa recommendation for EMB in cases of unexplained cardiomyopathy in children. [19]

\section{Transport considerations}

\subsection{Triage}

Pediatric patients with symptomatic myocarditis should be admitted to a pediatric tertiary care center. Klugman et al. reported that in their cohort of pediatric myocarditis patients $45 \%$ of patients required milrinone, 35\% needed epinephrine, and $25 \%$ were supported with mechanical ventilation. Extracorporeal membrane oxygenation was needed in $7 \%$ of patients, and cardiac transplantation in 5\%. [9] When triaging the patient, consideration should be given to the fact that any patient requiring the use of blood pressure support in the setting of acute myocarditis may quickly deteriorate and need mechanical cardiovascular support. Extracorporeal membrane oxygenation (ECMO) support is now increasingly viewed as optimal supportive therapy in anticipation of full cardiac recovery. [20] In larger children, a ventricular assist device (VAD) has also been used to support ventricular function during acute illness. In a previously published paper reporting the transport a series of children with myocarditis, there were five out of ten patients who required ECMO. Among those five patients there were three survivors. [15] In another retrospective review of 36 cases of histologically confirmed myocarditis ECMO was used in 4 patients (11\%). [21]

\subsection{Transport}

It has been estimated that fewer than ten percent of hospitals with intensive care unit beds have pediatric critical care beds. [22], [23] Therefore, pediatric admission to a tertiary intensive care unit frequently requires patient transport. Though emergency medical service teams are trained in basic pediatric resuscitation and stabilization, often times they do not have the breadth of experience or advanced training which would provide for the safest transport of the critically ill child. The use of a critical care transport teams on the other hand is strongly associated with decreased complication rates. [24]- [27] In particular for pediatric patients, the chance of an unplanned airway or cardiovascular event was 22 times greater when a critical care transport team was not used. [24] In any population of patients with a high risk for cardiopulmonary deterioration, consideration must be given to balancing the potential benefit 
of using a critical care transport team and the risk of holding the patient in the emergency department for a longer time period until the specialty team is available.

For the above reasons, patients who present with symptomatic myocarditis are best transported to a tertiary care center with a critical care transport team. These patients are at high risk for deteriorating during transport, and often require urgent interventions upon arrival at the receiving hospital. Helicopter transport may be faster than ground transport, although this is not always true in urban environments or if the involved facilities do not have an on-site helipad. [28] Helicopter transport guidelines have identified pediatric patients with symptomatic myocarditis as appropriate candidates for helicopter transport. [29] While an efficient mode of transport, medical helicopters have maximum distance limitations. There are also strict weather and altitude limitations to helicopter transport, which may affect ground and fixed wing transport to a lesser degree. A patient requiring frequent assessment or interventions may be challenging to care for in a helicopter due to noise, lack of space making access to the patient challenging and turbulence in flight. Additionally in a helicopter, and certainly in a fixed wing vehicle, it may be more difficult to divert to a different receiving facility should the patient become acutely unstable for transport. There is no evidence looking at pediatric myocarditis and ideal modes of transport. Data from adult patients shows that there are conflicting reports about the efficacy of different modes of transport, specifically helicopter versus ground transport. In 2012 a retrospective cohort study showed that among patients with major trauma admitted to level I or level II trauma centers, transport by helicopter compared with ground services was associated with improved survival to hospital discharge. [30] While there are earlier studies in agreement with these findings, other studies in the adult population have failed to show a benefit of helicopter transport. [31]- [34]

In summary, choosing a team and mode of transport for a patient is complex. There are many factors influencing decision-making surrounding patient transport. The medical team should consider the patient's anticipated medical needs and the risks of destabilization during transport, the urgency of the treatments needed at the receiving facility, transport logistics such as altitude, weather and distance, and the team availability and experience. [35]

\subsection{Treatment}

There are currently no specific therapies for acute fulminant myocarditis. The mainstay of therapy is supportive care to maintain cardiac output including mechanical ventilation, inotropic support and, if tolerated, afterload reduction and diuresis. For transport purposes intubation, ventilation and inotropic support play a larger role than other support strategies. In adult populations there have historically been more options for ventricular assist devices. However, pediatric assist devices have been successfully developed. In a recent study of the Excor Pediatric ventricular assist device (Berlin Heart), Fraser et al demonstrated that survival rates for patients awaiting heart transplant were significantly higher with the ventricular assist device than with ECMO. [36] This data is not specific for myocarditis, but is promising that assist devices can be effectively used in the pediatric population. Currently, the majority of patients with refractory cardiogenic shock and/or severe respiratory failure will likely require ECMO for ongoing support. 


\subsubsection{Intubation and sedation}

In patients with evidence of pulmonary edema the risk of worsening hypoxemia and potential for respiratory acidosis is concerning, as neither would be well tolerated from a cardiac standpoint. As respiratory demands increase to compensate for these issues, the oxygen consumption of the respiratory muscles can increase up to eightfold. [37] Intubation and mechanical ventilation will reduce respiratory muscle oxygen consumption, and thus overall myocardial oxygen demand. [37] The risks of the induction for intubation should be carefully weighed against these benefits, but declining status may force a clinician to proceed with endotracheal intubation prior to transport.

In general, positive pressure ventilation reduces left ventricular wall tension and left ventricular afterload, and therefore may improve cardiac output by this mechanism. However, other cardiopulmonary interactions associated with intubation and positive pressure ventilation may precipitate low cardiac output or cardiac arrest in a patient with biventricular failure. Those potentially harmful interactions include cessation of right sided venous return during the transition from spontaneous breathing to positive pressure ventilation, and systemic vasodilation and negative inotropy induced by medication used for induction of anesthesia. If possible, it is important to ensure that the patient is euvolemic prior to induction to preserve right ventricular preload upon initiation of positive pressure ventilation. It is also advisable to have an inotropic agent either initiated or prepared to infuse to support biventricular function. [38]The choice of specific induction agents is less important than recognizing that patients in failure will likely have limited contractile reserve, will be relatively preload dependent and will not respond well to rapid changes in afterload. [39] The choice of the appropriate medication for induction of anesthesia for intubation is important. Any agent may precipitate vasodilation and cardiac depression. Etomidate is well-known for a low rate of adverse hemodynamic effects, and the direct sympathomimetic effects of ketamine may be particularly beneficial in shock states. [40] Carefully titrated low-dose fentanyl may also provide appropriate levels of sedation and analgesia with a more favorable cardiac profile. Midazolam, propofol, and barbiturates are all likely to trigger hypotension at induction doses and should therefore be avoided. Atropine premedication may be considered in pediatric patients with bradycardia, though many patients with myocarditis are tachycardic on presentation. [38]

The adverse hemodynamic effects of positive pressure ventilation on right sided venous return may be ameliorated by using a strategy to minimize mean airway pressure, thus reducing intrathoracic pressure. This includes avoiding lung hyperinflation, minimizing peak inspiratory pressures, the use of short inspiratory times and adequate expiratory times and conservative use of positive end-expiratory pressure (PEEP). While PEEP may be helpful in managing pulmonary edema and hypoxemia, it should be used with caution as it may lead to decreased right ventricular preload and increased right ventricular afterload.

\subsubsection{Rate control}

Both tachycardia and bradycardia can pose risks to a pediatric patient in acute heart failure. Arrhythmias must be quickly recognized and treated. Transcutaneous pacing has been 
recognized as an easy, safe, and effective temporary measure of rate control but may require sedation and likely requires analgesia in the pediatric patient. [41]- [44] As mentioned, administering sedation in a pediatric patient with myocarditis and cardiovascular compromise could lead to further hemodynamic instability. Initiation of catecholamines such as dopamine may provide benefit in patients with complete heart block by increasing the ventricular escape rate to improve systemic perfusion in transport and should be considered before initiation of transcutaneous pacing in hemodynamically stable patients. However, when using such agents care should be taken not to acutely increase left ventricular afterload.

\subsubsection{Afterload reduction}

Management of heart failure should be employed if the patient can tolerate diuresis and afterload reduction, but is probably not advisable in the acute setting. Ideally this management would include diuretics to lower filling pressures and angiotensin-converting enzyme (ACE) inhibitors to reduce systemic vascular resistance and left ventricular afterload. Beta-blockade may be used as well, however the only randomized controlled trial of beta-blockade for treatment of pediatric heart failure failed to demonstrate a benefit. [45] Furthermore using a beta-blocker in the acute setting may complicate resuscitation efforts should a patient have critically compromised output or lose circulation altogether. In patients with significant dysfunction and diminished cardiac output systemic inodilators such as milrinone, are often useful if tolerated. Due to the risk of systemic hypotension and some risk of worsening myocardial dysfunction these interventions are best started in a tertiary care setting, not during transport.

\subsubsection{Levosimendan}

Levosimendan is a positive ionotrope and functions by binding to cardiac troponin $C$ to increase calcium sensitivity of myocytes. It also has vasodilatory effects in arterial, venous and coronary vasculature, which leads to afterload reduction and better matching of myocardial oxygen demand. [46]- [49] Therefore despite improving ventricular function, levosimendan does not significantly increase myocardial oxygen demand. Levosimendan is currently not FDA approved, so there is no collective experience with it the US centers. There are case reports of levosimendan being used successfully in both adult and pediatric myocarditis. [50]- [52] However, there are no larger, prospective studies to provide adequate evidence for routine use at this point. It remains unclear what potential benefit this drug would have in critical care transport.

\subsubsection{IVIG}

The benefit of immune modulation remains controversial, and is not usually an adjunct to consider during acute transport management. Intravenous immunoglobulins (IVIG) are the most commonly used immune modulator in myocarditis. Drucker et al. showed a statistically significant improvement in survival in pediatric patients treated with IVIG. [53] However McNamara conducted a randomized control trial in adults and failed to show any difference in survival among those treated with IVIG. [54] The data on the use of immunosuppressive 
agents such as prednisone, azathioprine and cyclosporine is not yet convincing. When the existing data was examined in a meta-analysis, Hia et al were not able to find statistical significance for improved outcomes. [55] That said, many centers currently use IVIG in the treatment of myocarditis and in certain cases immunosuppressive therapy may improve outcomes. [9], [56]

\subsubsection{Mechanical support}

In severe cases of cardiogenic shock patients may require rescue with veno-arterial (VA) ECMO or ventricular assist devices (VADs). Veno-venous (VV) ECMO is typically reserved for patients with predominant pulmonary failure. Whether requiring ECMO or VAD support, patients are best cared for in tertiary care centers with established ECMO programs.

VA-ECMO should be considered in patients with myocarditis only once routine supportive therapies have failed. [57], [58] While potentially life-sustaining in these cases, ECMO is not without risk. There is significant chance for hemorrhage, infectious complications and vascular injury during cannulation. There is also a risk of cerebral and coronary hypoxia and stroke. Less common, but potentially life-threatening are thrombotic events. Another complicating issue, which may ultimately compromise ventricular recovery, is left atrial hypertension secondary to poor ventricular function and decreased ejection while on ECMO. Left atrial hypertension can result in increased left ventricular end-diastolic pressure, subendocardial ischemia and pulmonary edema. There is no consensus on indications or technique for left atrial decompression, but it has been shown to relieve pulmonary edema and improve hemodynamics in one study. [59]

In experienced centers, ECMO is often successfully employed as a short-term rescue therapy for refractory cardiopulmonary failure. Though there is extensive experience with pediatric $\mathrm{ECMO}$, in addition to potential complications there are also other significant limitations: need for sedation, lack of mobility, and relatively short lifespan of the circuit. In cases where failure is more chronic, or transplant is needed, a VAD may be a more appropriate intervention. VADs are available as right (RVAD), left (LVAD) and bi-ventricular (BiVAD) devices. They have been used for ventricular recovery, destination devices and as bridges to heart transplant. A recent prospective, single-group pediatric trial showed that survival rates to transplant were significantly higher with the ventricular assist device than with ECMO. [36] Complications of assist devices are significant and similar to ECMO, including bleeding, stroke, infection and thrombotic events.

\subsubsection{Special consideration: ECMO on transport}

Pediatric ECMO is offered in many centers worldwide [60], and increasingly ECMO centers are confronted with the request to transport a patient on ECMO. A few centers in the United States and in Europe reported these transports in the literature. [61]- [67] One group reported the successful transport of 68 children on ECMO, traveling a distance between eight and 7500 miles. Overall ECMO survival was comparable with in-house survival on 
ECMO at the same institution. More importantly, no deaths occurred during ECMO transport. [66]

Bringing an ECMO team to a referring facility to place an unstable patient on extracorporeal support and then transport the patient back to a tertiary care center on ECMO has been suggested and, in a few cases, successfully completed. The logistics of providing such a service are very complicated. Based on military data, Coppola and colleagues reported that the ECMO transport team consists of 10-15 staff members, including a mission commander, a pediatric intensivist, a pediatric cardiologist, a pediatric surgeon, two to three ECMO specialists, nurses and respiratory therapists [66]. A civilian team reported using a team consisting of two nurses, two ECLS specialists, an attending physician, and a resident. [67] ECMO transports to date have been completed in ground, fixed-wing, and rotor-wing vehicles. The complexity of ECMO transport warrants careful discussion about feasibility and resource utilization, but may be successfully accomplished. That said, early referral to an ECMO center while the patient may be safely transported without ECMO is the preferred option.

\section{Conclusions}

Myocarditis presents with a broad range of relatively non-specific symptoms and for that reason is difficult to diagnose, but must remain on the list of differential diagnoses for any child presenting with acute heart failure or other signs of cardiac deterioration. Acute fulminant myocarditis is life-threatening and requires careful, proactive management. When treating the pediatric patient with acute fulminant myocarditis clinicians should consider the benefits of intubation, inotropic infusions, and transcutaneous pacing as temporizing measures especially during the transport phase, recognizing that any of those interventions can lead to further deterioration of the patient if not performed with great caution. Prompt and safe transport to a pediatric tertiary care center should be ensured. The option of early management with ECMO or other assist devices seems beneficial and should be considered when making triage decisions.

\section{Author details}

Jordan S. Rettig ${ }^{1}$ and Gerhard K. Wolf ${ }^{*}$

*Address all correspondence to: gerhard.wolf@childrens.harvard.edu

1 Division of Cardiac Critical Care, Department of Cardiology, Perioperative and Pain Medicine, Boston Children's Hospital, Harvard Medical School, Boston, Massachusetts, USA

2 Division of Critical Care Medicine, Department of Anesthesiology, Perioperative and Pain Medicine, Boston Children's Hospital, Harvard Medical School, Boston, Massachusetts, USA 


\section{References}

[1] Liu, R, \& Schulteiss, H. Myocarditis. In: Libby P, DL M, O’Bonow R, DP Z, eds. Brunwald's Heart Disease: A Textbook of Cardiovascular Medicine. 8th ed. Philadelphia: Saunders Elsevier; (2007). , 2007, 1775-85.

[2] Kearney, M. T, Cotton, J. M, Richardson, P. J, \& Shah, A. M. Viral myocarditis and dilated cardiomyopathy: mechanisms, manifestations, and management. Postgrad Med J (2001). , 77, 4-10.

[3] Vashist, S, \& Singh, G. K. Acute myocarditis in children: current concepts and management. Curr Treat Options Cardiovasc Med (2009). , 11, 383-91.

[4] Robinson, J, Hartling, L, Vandermeer, B, Crumley, E, \& Klassen, T. P. Intravenous immunoglobulin for presumed viral myocarditis in children and adults. Cochrane Database Syst Rev (2005). CD004370.

[5] Weber, M. A, Ashworth, M. T, Risdon, R. A, Malone, M, Burch, M, \& Sebire, N. J. Clinicopathological features of paediatric deaths due to myocarditis: an autopsy series. Arch Dis Child (2008). , 93, 594-8.

[6] Shekerdemian, L, \& Bohn, D. Acute viral myocarditis: Epidemiology and pathophysiology.. Pediatr Crit Care Med (2006). SS7., 2.

[7] Sugent, A, \& Daunbeney, P. P C. The epidemiology of childhood cardiomyopathy in Australia. N Engl J Med (2003). , 348, 1639-46.

[8] Lipshultz, S, Sleeper, L, \& Towbin, J. The incidence of pediatric cardiomyopathy in two regions of the United States. N Engl J Med (2003). , 348, 1647-55.

[9] Klugman, D, Berger, J. T, Sable, C. A, He, J, Khandelwal, S. G, \& Slonim, A. D. Pediatric patients hospitalized with myocarditis: a multi-institutional analysis. Pediatr Cardiol (2010). , 31, 222-8.

[10] Lieberman, E. B, Hutchins, G. M, Herskowitz, A, Rose, N. R, \& Baughman, K. L. Clinicopathologic description of myocarditis. J Am Coll Cardiol (1991). , 18, 1617-26.

[11] Amabile, N, Fraisse, A, Bouvenot, J, Chetaille, P, \& Ovaert, C. Outcome of acute fulminant myocarditis in children. Heart (2006). , 92, 1269-73.

[12] (Maron BJ, Doerer JJ, Haas TS, Tierney DM, Mueller FO. Sudden deaths in young competitive athletes: analysis of 1866 deaths in the United States, 1980-2006. Circulation 2009;119:1085-92). 119, 1085-92.

[13] Nugent, A. W, Daubeney, P. E, Chondros, P, et al. The epidemiology of childhood cardiomyopathy in Australia. N Engl J Med (2003). , 348, 1639-46. 
[14] Freedman, S. B, Haladyn, J. K, Floh, A, Kirsh, J. A, Taylor, G, \& Thull-freedman, J. Pediatric myocarditis: emergency department clinical findings and diagnostic evaluation. Pediatrics (2007). , 120, 1278-85.

[15] Wolf, G. K, Frakes, M. A, Gallagher, M, Allan, C. K, \& Wedel, S. K. Management of suspected myocarditis during critical-care transport. Pediatr Emerg Care (2010). , 26, 512-7.

[16] Checchia, P, \& Kulik, T. Acute viral myocarditis. Pediatr Crit Care Med (2006). SS11., 8.

[17] Smith, S. C, Ladenson, J. H, Mason, J. W, \& Jaffe, A. S. Elevations of cardiac troponin I associated with myocarditis. Experimental and clinical correlates. Circulation (1997). , 95, 163-8.

[18] Soongswang, J, Durongpisitkul, K, Nana, A, et al. Cardiac troponin T: a marker in the diagnosis of acute myocarditis in children. Pediatr Cardiol (2005). , 26, 45-9.

[19] Cooper, L. T, Baughman, K. L, Feldman, A. M, et al. The role of endomyocardial biopsy in the management of cardiovascular disease: a scientific statement from the American Heart Association, the American College of Cardiology, and the European Society of Cardiology. Circulation (2007). , 116, 2216-33.

[20] Bohn, D, Macrae, D, \& Chang, A. Acute viral myocarditis: mechanical circulatory support. Pediatr Crit Care Med (2006). SS4., 21.

[21] Lee, K. J, Mccrindle, B. W, Bohn, D. J, et al. Clinical outcomes of acute myocarditis in childhood. Heart (1999). , 82, 226-33.

[22] Randolph, A, Gonzales, C, Crotellini, L, \& Yeh, T. Growth of pediatric intensive care units in the United States. Pediatrics (2004). , 144, 792-8.

[23] Guss, C, Brower, R, \& Hudson, L. Indcidence of acute lung injury in the United States. Crit Care Med (2003). , 31, 1607-11.

[24] Orr, R. Unplanned events in pediatric critical care transport. Pediatrics (1999). S687.

[25] Edge, W, Kanter, R, \& Weigle, C. Reduction of morbidity in interhospital transport by specialized pediatric staff. Crit Care Med (1994). , 22, 1186-91.

[26] Frakes, M. Flight team management of in-place endotracheal tubes. Air Med J (2002). , 21, 29-31.

[27] Gebermichael, M. Interhospital transport of the extremely ill patient. Crit Care Med (2000). , 28, 79-85.

[28] Svenson, J, Connor, O, \& Lindsay, J. B. A comparison of air versus ground transport times for interfacility transfers in a regional referral system. Air Med J (2005). , 24, $70-2$. 
[29] Thompson, D, \& Thomas, S. Guidelines for air medical dispatch. Prehosp Emerg Care (2003). , 7, 265-71.

[30] Galvagno, S. M. Jr., Haut ER, Zafar SN, et al. Association between helicopter vs ground emergency medical services and survival for adults with major trauma. JAMA (2012). , 307, 1602-10.

[31] Rose, M. K, Cummings, G. R, Rodning, C. B, Brevard, S. B, \& Gonzalez, R. P. Is helicopter evacuation effective in rural trauma transport? Am Surg (2012). , 78, 794-7.

[32] Shepherd, M. V, Trethewy, C. E, Kennedy, J, \& Davis, L. Helicopter use in rural trauma. Emerg Med Australas (2008). , 20, 494-9.

[33] Stewart, K. E, Cowan, L. D, Thompson, D. M, Sacra, J. C, \& Albrecht, R. Association of direct helicopter versus ground transport and in-hospital mortality in trauma patients: a propensity score analysis. Acad Emerg Med (2011). , 18, 1208-16.

[34] Butler, D. P, Anwar, I, \& Willett, K. Is it the H or the EMS in HEMS that has an impact on trauma patient mortality? A systematic review of the evidence. Emerg Med J (2010). , 27, 692-701.

[35] Blumen, I, Corbett, P, \& Krost, W. Considerations in vehicle selection for patient transport. In: Blumen I, ed. Principles and direction of air medical transport. Salt Lake City: Air Medical Physician's Association; (2006).

[36] Fraser, C. D. Jr., Jaquiss RD, Rosenthal DN, et al. Prospective trial of a pediatric ventricular assist device. N Engl J Med (2012). , 367, 532-41.

[37] Mason, R, Murray, E, Broaddus, V, \& Nadel, J. Murray and Nadel's Textbook of Respiratory Medicine. 4th ed. Philadelphia: Saunders; (2005).

[38] Miller, R. Anesthesia. 6th ed. New York: Churchill Livingstone; (2005).

[39] DiNardo JAZvara DA. Anesthesia for cardiac surgery. 3rd ed. Malden, Mass.: Blackwell Pub.; (2008).

[40] Bergen, J, \& Smith, D. A review of etomidate for rapid sequence intubation in the emergency department. J Emerg Med (1997). , 15, 221-30.

[41] Altamaura, G, \& Toscano, S. LoBianco F, Catalno F, Pistolese M. Emergency cardiac pacing for severe bradycardia. Pacing Clin Electrophysiol (1990). , 13, 2038-43.

[42] Madsen, J, Meiborn, J, Videbak, R, Pedersen, F, \& Grande, P. Transcutaneous pacing: Experience with the Zoll noninvasive temporary pacemaker. Am Heart J (1988). , 116, 7-10.

[43] Zoll, P, \& Zoll, R. Noninvasive temporary cardiac stimulation. Crit Care Med (1985). , $13,925-6$. 
[44] Belano, M, Hesslein, P, Finlay, C, Faerron-angel, J, Williams, W, \& Rowe, R. Noninvasive transcutaneous pacing in children. Pacing Clin Electrophysiol (1987). , 10, 1262-70.

[45] Shaddy, R. E, Boucek, M. M, Hsu, D. T, et al. Carvedilol for children and adolescents with heart failure: a randomized controlled trial. JAMA (2007). , 298, 1171-9.

[46] Parissis, J. T, Adamopoulos, S, Antoniades, C, et al. Effects of levosimendan on circulating pro-inflammatory cytokines and soluble apoptosis mediators in patients with decompensated advanced heart failure. Am J Cardiol (2004). , 93, 1309-12.

[47] Parissis, J. T, \& Filippatos, G. Levosimendan in viral myocarditis: not only an inodilator but also a cardioprotector? Eur J Clin Invest (2009). , 39, 839-40.

[48] Pollesello, P, \& Papp, Z. The cardioprotective effects of levosimendan: preclinical and clinical evidence. J Cardiovasc Pharmacol (2007). , 50, 257-63.

[49] Antila, S, Sundberg, S, \& Lehtonen, L. A. Clinical pharmacology of levosimendan. Clin Pharmacokinet (2007). , 46, 535-52.

[50] Schweigmann, U, Velik-salchner, C, Kilo, J, \& Schermer, E. How mechanical circulatory support helps not to need it--new strategies in pediatric heart failure. Artif Organs (2011). , 35, 1105-9.

[51] Busani, S, Pasetto, A, Ligabue, G, Malavasi, V, Lugli, R, \& Girardis, M. Levosimendan in a case of severe peri-myocarditis associated with influenza A/H1N1 virus. Br J Anaesth (2012). , 109, 1011-3.

[52] Latva-hirvela, J, Kyto, V, Saraste, A, Vuorinen, T, Levijoki, J, \& Saukko, P. Effects of levosimendan in experimental acute coxsackievirus myocarditis. Eur J Clin Invest (2009). , 39, 876-82.

[53] Drucker, N. A, Colan, S. D, Lewis, A. B, et al. Gamma-globulin treatment of acute myocarditis in the pediatric population. Circulation (1994). , 89, 252-7.

[54] Mcnamara, D. M, Holubkov, R, Starling, R. C, et al. Controlled trial of intravenous immune globulin in recent-onset dilated cardiomyopathy. Circulation (2001). , 103, 2254-9.

[55] Hia, C. P, Yip, W. C, Tai, B. C, \& Quek, S. C. Immunosuppressive therapy in acute myocarditis: an 18 year systematic review. Arch Dis Child (2004). , 89, 580-4.

[56] Camargo, P. R, \& Snitcowsky, R. da Luz PL, et al. Favorable effects of immunosuppressive therapy in children with dilated cardiomyopathy and active myocarditis. Pediatr Cardiol (1995). , 16, 61-8.

[57] Chen, Y. S, Wang, M. J, Chou, N. K, et al. Rescue for acute myocarditis with shock by extracorporeal membrane oxygenation. Ann Thorac Surg (1999). , 68, 2220-4. 
[58] Duncan, B. W, Bohn, D. J, Atz, A. M, French, J. W, Laussen, P. C, \& Wessel, D. L. Mechanical circulatory support for the treatment of children with acute fulminant myocarditis. J Thorac Cardiovasc Surg (2001). , 122, 440-8.

[59] Aiyagari, R. M, Rocchini, A. P, Remenapp, R. T, \& Graziano, J. N. Decompression of the left atrium during extracorporeal membrane oxygenation using a transseptal cannula incorporated into the circuit. Crit Care Med (2006). , 34, 2603-6.

[60] The ELSO Registry(2009). Accessed 2009, at http://www.elso.med.umich.edu/Registry.htm.)

[61] Heulitt, M, Taylor, B, \& Faulkner, S. Interhospital transport of neonatal patients on extracorporeal membrane oxygenation: mobile-ECMO. Pediatrics (1995). , 95, 562-6.

[62] Rossaint, R, Pappert, D, \& Gerlach, H. Extracorporeal membrane oxygenation for transport of hypoxemic patients with severe ARDS. Br J Anesth (1997). , 78, 241-6.

[63] Linden, V, Palmer, K, \& Reinhard, J. Interhospital transportation of patients with severe acute respiratory failure on extracorporeal membrane oxygenation- national and international experience. Intensive Care Med (2001). , 27, 1643-8.

[64] Foley, D, Pranikoff, T, \& Younger, J. A review of 100 patients transported on extracorporeal life support. ASAIO J (2002). , 48, 612-9.

[65] Huang, S, Chen, Y, \& Chi, N. Out-of-center extracorporeal membrane oxygenation for adult cardiogenic shock patients. Artif Organs (2006). , 30, 24-8.

[66] Coppola, C, Tyree, M, \& Larry, K. DiGeronimo R. A 22-year experience in global transport extracorporeal membrane oxygenation. J Pediatr Surg (2008). , 43, 46-52.

[67] Bowers, W, \& Wyrick, P. Extracorporeal life support: A transcontinental transport experience. Air Med J (2003). , 12, 7-11. 